\title{
Terrain Negotiation of a Compliant Biped Robot Driven by Antagonistic Artificial Muscles
}

\author{
Takashi Takuma, Koh Hosoda \\ Osaka University \\ 2-1 Yamadaoka, Suita, Osaka, Japan \\ \{takuma, hosoda\}@ams.eng.osaka-u.ac.jp
}

\begin{abstract}
Humans can negotiate various classes of terrain and realize adaptive bipedal walking, which is difficult for existing biped robots driven by electric motors. In this paper, a biped robot driven by antagonistic artificial muscles is developed that can negotiate several classes of terrain. The antagonistic muscles along with a simple control scheme can realize joint compliance without a time-delay. Not only does the compliance adapt the robot to terrain changes, but it also enables the robot to sense the terrain since the walking behavior is a result of the interaction between the dynamics of the robot body and the terrain. Experimental results demonstrate that the walking cycle changes according to joint compliance and the class of terrain. Utilizing the relationship between the two, the robot can regulate its walking cycle by changing its joint compliance. The compliance by such antagonistic muscles is a promising solution for realizing adaptive bipedal walking.
\end{abstract}

\section{Introduction}

Humans adjust their joint compliance by utilizing their antagonistic muscles to negotiate various classes of terrain [1]. Locomotion is then achieved by the interaction between body dynamics, including joint compliance, and the dynamics of the terrain [2]. On the other hand, almost all of the existing humanoid robots are equipped with electric motors and high-reduction gears $[3,4,5]$ and are designed to follow a desired trajectory despite the terrain. It is therefore difficult for these robots to negotiate various classes of terrain adaptively. Instead, they require elaborate torque/force feedback to realize joint compliance, which often results in a large time-delay.

Some researchers have focused on joint compliance for adaptive bipedal walking in the context of Passive Dynamic Walking [6]. Kuo et al. [7] and Van der Linde et al. [8] investigated the relationship between hip compliance and walking behavior using numerical simulations. Wisse et al. adopted antagonistic artificial muscles for robust biped walking [9]. However, they only conducted experiments on a uniform surface and did not address the contribution of joint compliance to the negotiation of various classes of terrain.

In this paper, a bipedal robot driven by antagonistic artificial muscles is built that can negotiate several classes of terrain. The muscles along with a simple feedforward controller can realize joint compliance without a time-delay. The walking behavior is generated by the interaction between the robot dynamics and the terrain. Therefore, the walker is able to realize adaptive locomotion and sense the terrain as well. The walker is also able to change its walking behavior by tuning its joint compliance. Experimental results show that the robot walks on two types of terrain with different joint compliance, and that the walking cycle changes according to the compliance and the class of the terrain. This relationship between the walking cycle and the joint compliance is utilized to design a feedback controller that regulates the walking cycle.

The rest of the paper is organized as follows. First, the specification of the passive walker Que-Kaku driven by pneumatic actuators is detailed in Section 2. Second, a feedforward controller employed to achieve walking is introduced in Section 3. Then in Section 4, it is shown that the walking behavior changes according to the class of the terrain, and the relationship between the walking cycle, compliance and the terrain is developed. Utilizing this relationship, a feedback controller to regulate the walking cycle is designed. Section 5 details an experiment in which the walking cycle is regulated by the feedback controller.

\section{A 2D Biped Walker with Pneumatic Muscles}

\subsection{Compliant Joint Mechanism}

In order to drive the hip joint and keep the joint compliant, a mechanism by which the joint is driven by the agonistic and antagonistic McKibben pneumatic actuators [8, 9] is employed. Figure 1 shows the architecture 
of a planar biped walker driven by agonistic and antagonistic McKibben pneumatic muscles. When air is supplied to the muscle, the muscle contracts, thereby generating a tensile force. The muscle has a nonlinear springy nature, and the compliance increases according to the increase in pressure.

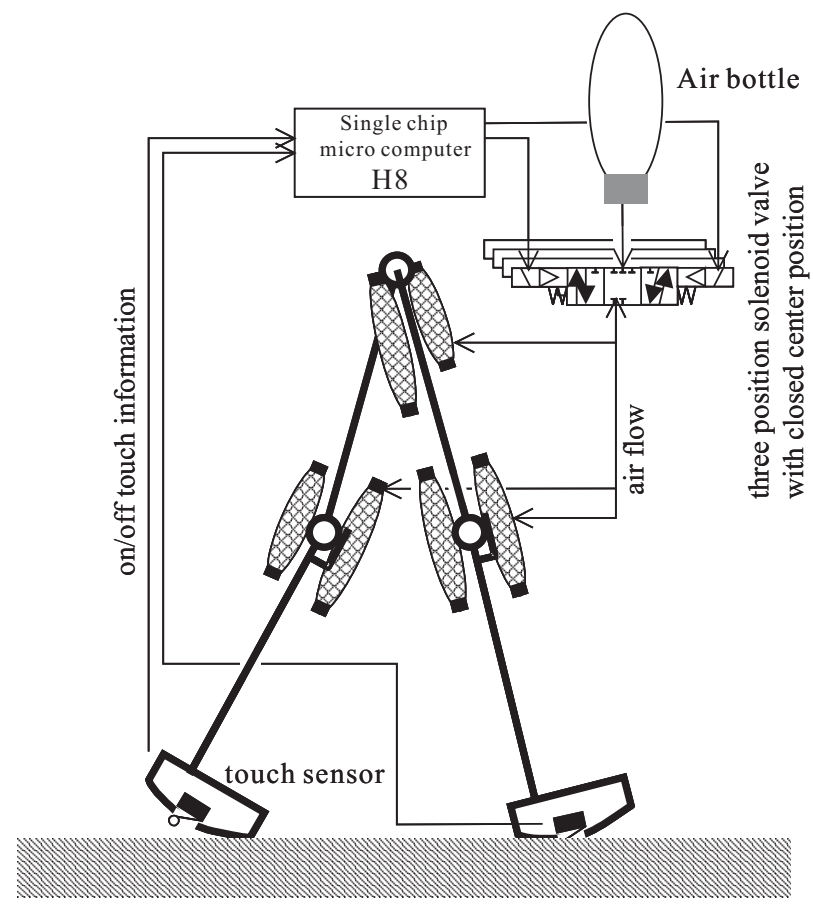

Figure 1: Architecture of a planar biped walker driven by antagonistic pairs of McKibben pneumatic muscles. The main feature of the walker is that it is equipped with the three-position solenoid valve with a closed center position that enables the robot to control the joint compliance.

In order that the joint compliance is continuously changeable, three-position solenoid valves are utilized. The valve takes three positions: supplying air from the source, expelling air to the atmosphere, and closing to quit both supplying and expelling. Figure 2 shows the pressure of the actuator increasing when the compressed air $(0.6[\mathrm{MPa}])$ is fulfilled into the actuator. This figure shows that it takes about 0.8 [s] to reach $0.6[\mathrm{MPa}]$ (solid line). On the other hand, the computer that operates air valves sends the signal to open / close valve at arbitral time, and the response time of the valve is less than 0.03 [s]. Therefore, it is possible to realize continuous change of the pressure by tuning the duration to open / close valve. In Figure 2, the pressure is changed by tuning the duration to supply the air. The broken lines in the Figure 2 show the pressure when the valve is open for some period $(0.1,0.15$, and $0.2[\mathrm{~s}])$ and then close. When the duration is shorter, the pressure after closing the valve is lower and vice versa. Because the compliance is changed according to the pressure and the joint is driven by the antagonistic pneumatic muscles, the joint compliance is controlled by tuning the durations of both muscles. The difference between [9] is that [9] utilizes a two-position solenoid valve that either supplies or expels. Therefore, it cannot maintain certain compliance of the joint.

\subsection{Specification of 2D Biped Walker}

Figure 3 shows a picture of the real-world prototype "Que-kaku." All of the joints are driven by McKibben pneumatic muscles [10, 11] manufactured by the HITACHI Medical Corporation [12]. The length and radius (in shrinkage) of the muscle are $0.15[\mathrm{~m}]$ and $0.027[\mathrm{~m}]$, respectively. The prototype generates approximately $800[\mathrm{~N}]$ when the pressure in the inner tube is $0.7[\mathrm{MPa}]$.

The walker has three joints: two knees and one hip. Each joint is driven by antagonistic and agonistic pneumatic muscles. The height, width, and weight of the walker are $0.75[\mathrm{~m}], 0.35[\mathrm{~m}]$, and $5[\mathrm{~kg}]$, respectively. The walker has four legs to prevent sideways swaying. The inner and outer legs are connected to each other in pairs. The length and weight of the thigh are $0.3[\mathrm{~m}]$ and $2.16[\mathrm{~kg}]$, respectively, and the length and weight of the shank are $0.35[\mathrm{~m}]$ and $0.48[\mathrm{~kg}]$, respectively.

The robot is self-contained and has two $1.2[\mathrm{MPa}] \mathrm{CO}_{2}$ bottles with regulators as air sources, each of which weighs $0.7[\mathrm{~kg}]$, a microcomputer $(\mathrm{H} 8)$, and an electrical battery that weighs 0.1 [kg]. The robot has six sets of three-position solenoid valves (SMC corporation [13] SYJ3340-6M-M5) that weigh a total of $0.6[\mathrm{~kg}]$. The air pressure is maintained at $0.55[\mathrm{MPa}]$ by the regulator on each bottle and the regulated 


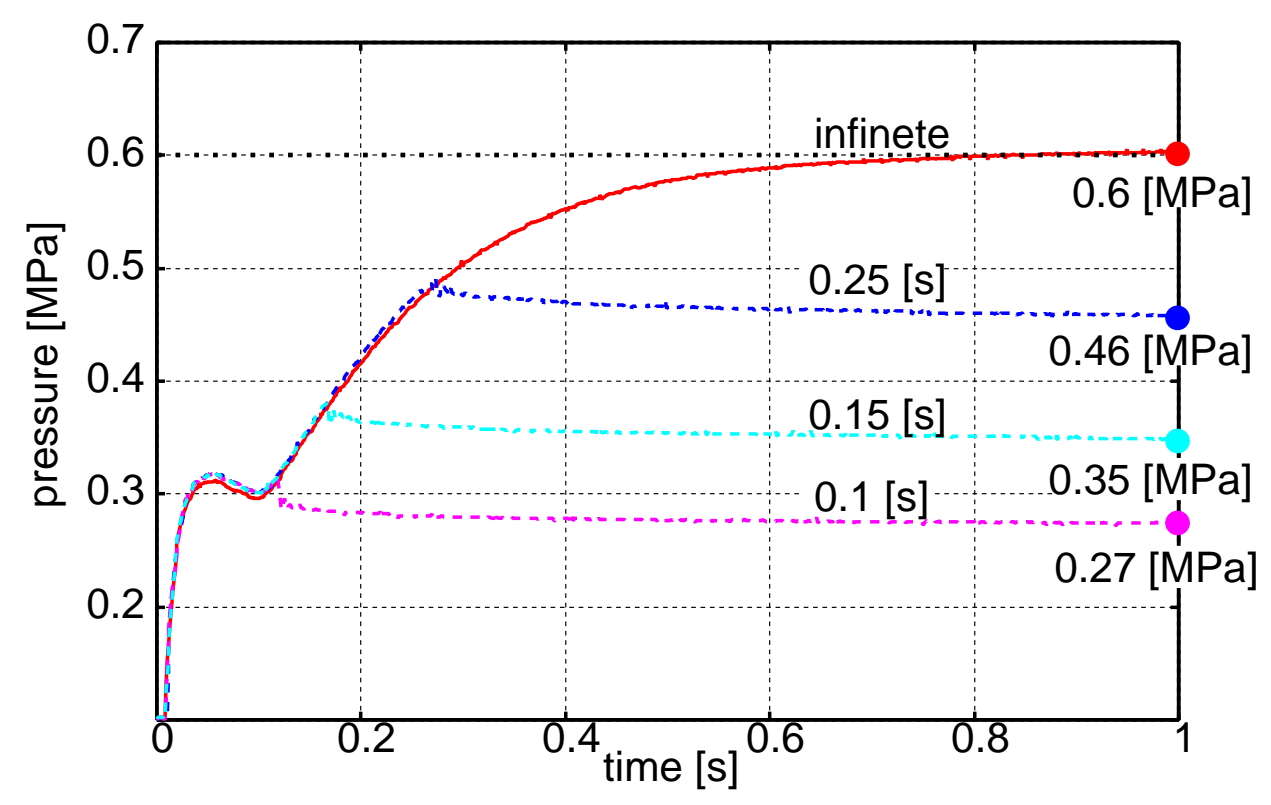

Figure 2: Air pressure increasing after opening supply valve. The air of $0.6[\mathrm{MPa}]$ is fulfilled for 0.8 [s] (solid line) while the valve is open or close at arbitral moment. The pressure is controlled by tuning the supply duration (broken line : closed at $0.1,0.15$, and $0.25[\mathrm{~s}]$ ).

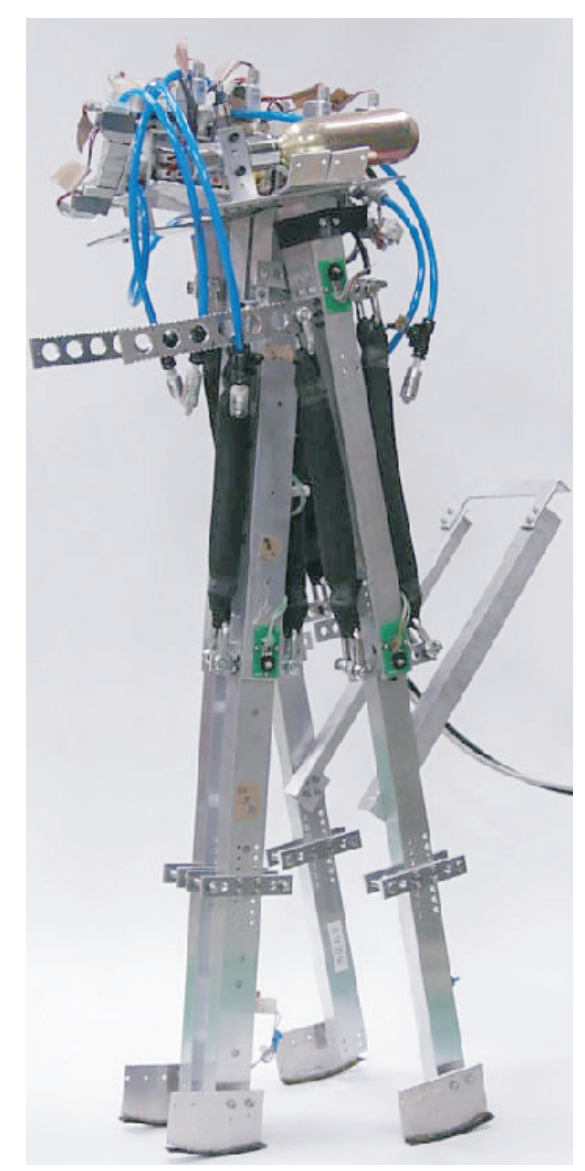

Figure 3: A 2D biped walker with McKibben pneumatic muscles. The robot is self-contained and has two air bottles with regulators, three-position air valves, a microcomputer board, and an electrical battery. 
air is supplied to the muscle by the valves. The feet have round soles with a curvature of 0.125 [m] and a length of $0.08[\mathrm{~m}]$. The robot has $\mathrm{ON} / \mathrm{OFF}$ switches that detect collisions with the ground (see Fig. 1). The $\mathrm{ON} / \mathrm{OFF}$ information is fed to the microcomputer. Then, depending on the received information, the computer outputs open/close commands to the solenoid valves.

\section{Feedforward Controller}

In order to walk on a flat plane with various hip compliances using a simple feedforward controller, the robot was designed with a specific center of mass (CoM) and radius of arced feet. The CoM and the arc radius are determined by trial and error. The relationship between CoM and the possibility to walk is shown in [14]. We also attached the feet with various radius of arc and adopted the specific radius to realize walking.

The feedforward controller determines the durations that the supply and expel valves are open and closed in order to drive the joint and to keep the joint compliant. The procedure for opening the valves is explained below. The timing chart to opening valves is shown in Figure 4:

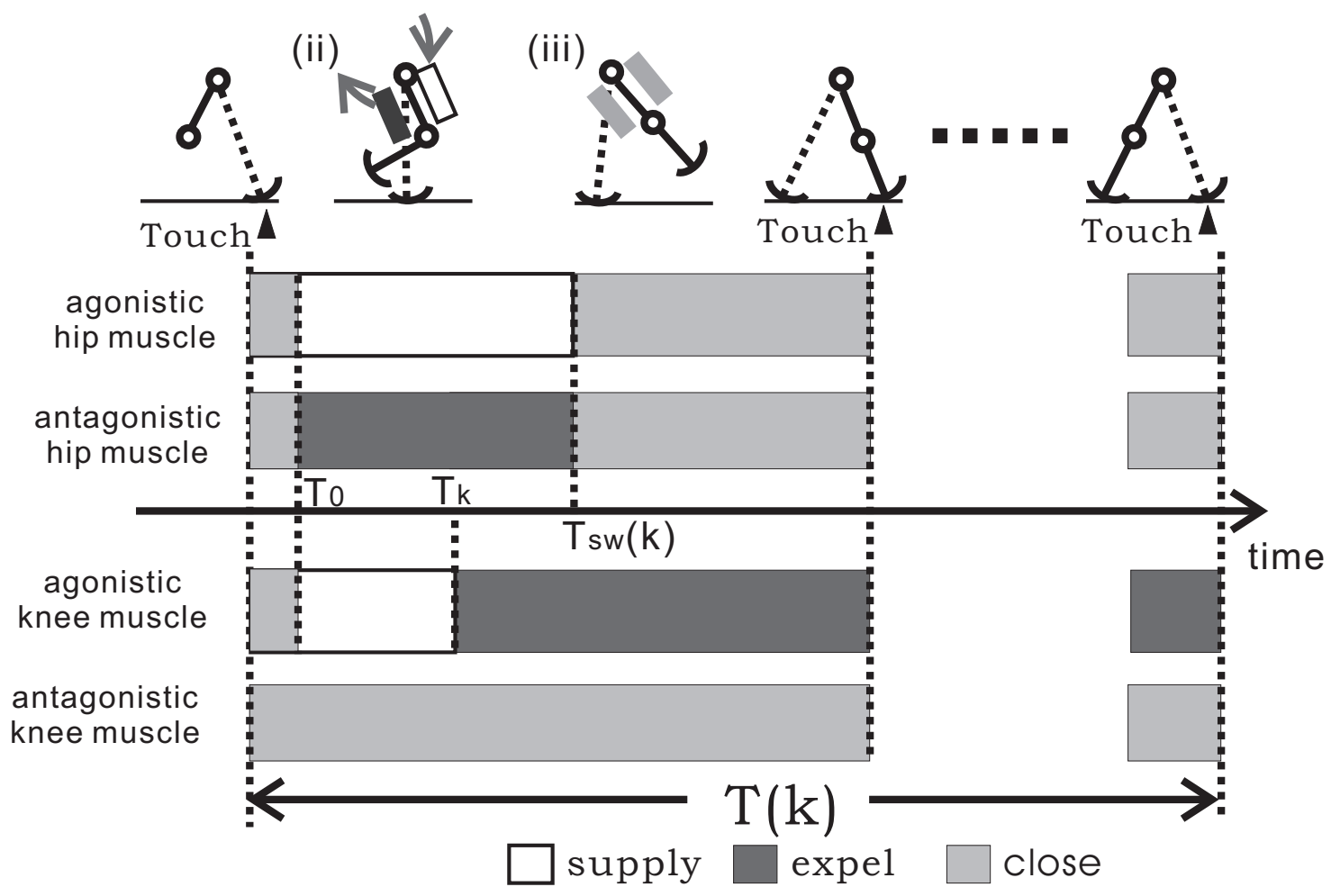

Figure 4: Feedforward controller: schematic explanation of when to actuate the muscles and when to relax them

(i) When the touch sensor on the swing leg's foot detects the landing, all valves are closed for $T_{0}$. While closing all valves, the hip joint and the whole body are driven passively.

(ii) After $T_{0}$, the air is supplied into the front hip muscle, which corresponds to the agonistic muscle, and the air is expelled from the back hip muscle, which corresponds to the antagonistic muscle. In this paper, $T_{s w}(k)$ is defined as the duration to supply or expel the air at the $k$-th step. At the same time, the air is supplied into the agonistic muscle of the swing leg's knee to avoid scuffing the foot for $T_{k}$, and then the air is expelled until the leg touches the ground. Some amount of air is supplied into antagonistic muscle of the swing leg's knee beforehand, therefore the knee bends again after the air in the agonistic knee muscle is expelled.

(iii) After $T_{0}+T_{s w}(k)$, all valves except the expel valve on the agonistic knee muscle are closed. While closing the valves, the hip joint maintains its compliance, and then the hip joint is driven passively. While the joint maintains its compliance, the whole body of the robot moves ballistically. After the swing leg landing is detected by the ON/OFF touching sensor, the sequence of the valve opening procedure returns to Step (i).

The walking cycle $T(k)$ is defined as the period from when a leg touches the ground to when the same leg touches the ground again. After test trials to achieve walking, walking parameters $T_{0}$ and $T_{k}$ were fixed at $T_{0}=0.032[\mathrm{~s}]$ and $T_{k}=0.3[\mathrm{~s}]$. The duration $T_{s w}(k)$ is a walking parameter that determines the hip joint 
compliance. In the trials, it was also found that the robot achieves walking when the walking parameter $T_{s w}(k)$ is within a certain range. This suggests that the robot can walk at various hip joint compliances.

\section{Terrain Negotiation Utilizing Compliant Joint}

Using the feedforward controller described in the previous section, it was observed that the robot could walk on a flat plane at an appropriate value of walking parameter $T_{s w}(k)$. It is also observed that the walking behavior, the walking cycle $T(k)$ in this paper, is influenced not only by the walking parameter $T_{s w}(k)$ shown in [15], but also by the terrain. The reason behind the influence of the terrain is that the emergence of the walking behavior partly depends on the properties of the contacting ground. Therefore, the walking behavior changes according to the terrain. This section investigates the influence on the walking cycle by the types of terrain. Then, a feedback controller is designed to regulate the walking cycle.

In this experiment, the robot walks on two types of floors: a linoleum floor (see Figure 5(a)) and a carpet floor (see Figure 5(b)). The linoleum is used for floors in many buildings, and it is harder than carpet. The carpet used in the experiment is a common commodity. The carpet was placed on top of the linoleum floor, as shown in Figure 5 (b). Each terrain has small ups and downs in places, and the gradient range is within 1 degree. It is hard to measure the quantitative property, such as stiffness and friction coefficient, of the terrain. The property of the physical terrain is also not uniform but different within some amount of range at each terrain. One of the claims in this paper is that the variation of the walking cycle shows the difference of the terrain. Therefore, it is thought that the quantitative properties of the terrain do not necessarily support to understand the terrain that affects the walking behavior.

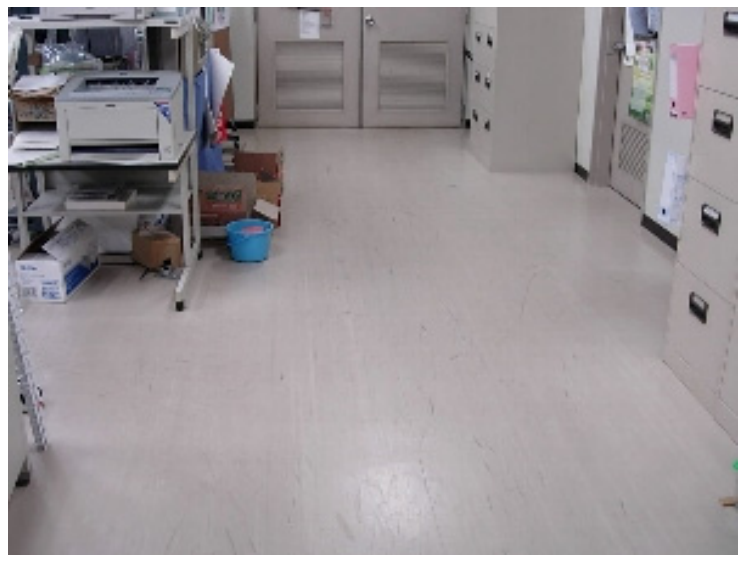

(a) Linoleum floor

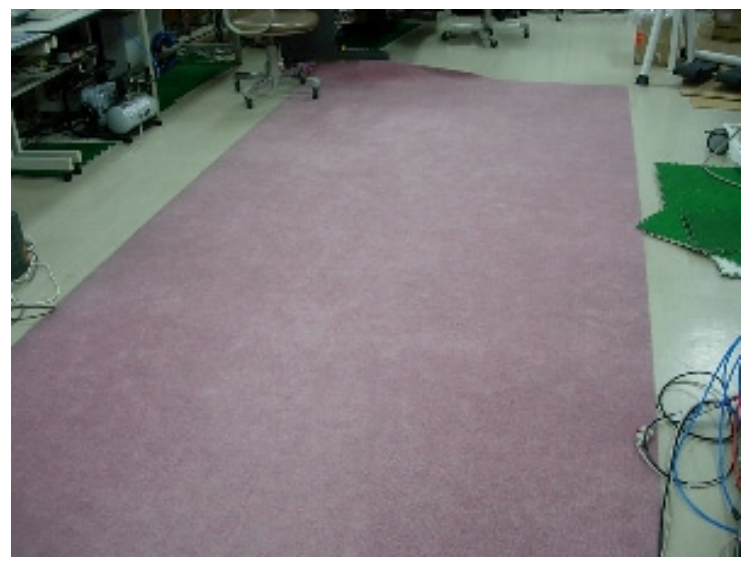

(b) Carpet floor

Figure 5: Two classes of terrain

Figure 6 shows the walking cycles when the robot walked on the linoleum floor (solid line) and on the carpet (broken line). The walking parameter $T_{s w}(k)$ was fixed at $0.24[\mathrm{~s}]$ in both cases. As mentioned above, each terrain has different gradient and property in places within some range. It can be explained that the distribution of walking cycle at each step was occurred by such an uneven terrain properties. Although the walking cycle is not even, these cycles are within some range, and they are evidently different. The walking cycle at linoleum floor is shorter than the case of carpet. Considering that the walking parameter $T_{s w}(k)$ was fixed in these two experiments, the difference between the walking cycles in the figure was caused by the differences in terrain conditions, such as the compliance and the friction. This experimental result shows that the robot developed different walking behaviors according to the existing floor conditions. By observing the difference, the classes of the terrain are estimated. Because the terrain and robot dynamics are too complex to be described as a formal model, the relationship between actuation (air valve opening duration) and walking behavior (the walking cycle) are investigated by real walking trials in the next section. 


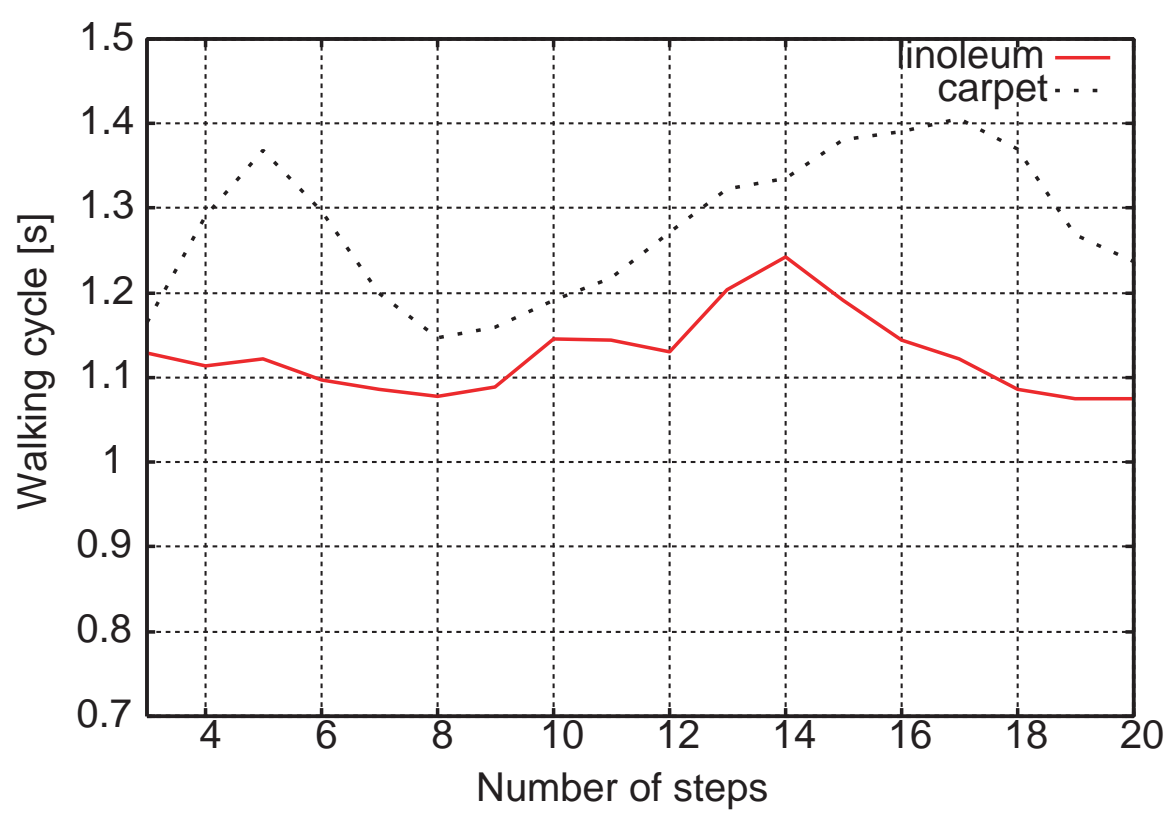

Figure 6: The walking cycles when the robot walked on the different ground coverings for the same walking parameter $T_{s w}(k)=0.24[\mathrm{~s}]$ after the 3 rd step

\section{$5 \quad$ Design of Step-by-step Feedback Controller}

\subsection{Feedback Controller Derived from Observed Relations}

In this section, the relationship between the valve opening duration and the walking cycle on various two classes of terrain is investigated. Then, a feedback controller is designed that can regulate the walking cycle utilizing the observed relationship. Figure 7 shows the relationship between $T_{s w}(k)$ and $T(k)$ on the linoleum floor (indicated by "+") and the carpet (indicated by " $\times$ "). The walking cycles were observed for parameter $T_{s w}(k)=0.18,0.2,0.22,0.24,0.26,0.28,0.3[\mathrm{~s}]$ on both floors. During the observation, the walking cycle was recorded after the robot shows a cyclic locomotion. In the figure, the "walking cycle" denotes the average period of 100 steps at each walking parameter. As shown in the previous experiments, the walking cycles are different with the same walking parameter on the different floors. In both cases, it is evident that there are positive correlations between the walking parameter and the walking cycle. It is also observed that the gradients of the relationships are almost same. The solid and broken lines in the figure are the approximated lines calculated by the least squares method when the robot walks on the carpet and linoleum floor, respectively. The approximated lines are, represented by $T_{c}\left(T_{s w}\right)$ when the robot walks on the carpet and $T_{l}\left(T_{s w}\right)$ when the robot walks on the linoleum floor, expressed as follows:

$$
\begin{array}{r}
T_{c}\left(T_{s w}\right)=1.57 T_{s w}+0.846 \\
T_{l}\left(T_{s w}\right)=1.63 T_{s w}+0.74
\end{array}
$$

From these relations, a PI feedback controller such as:

$$
T_{s w}(k)=T_{f f}-K_{p}\left(T(k)-T_{d e s}\right)-K_{i} \sum_{i}^{k}\left(T(i)-T_{d e s}\right),
$$

is proposed where $T_{f f}$ is a constant, $K_{p}, K_{i}$ are positive constants, and $T_{\text {des }}$ is a desired walking cycle. Utilizing the controller, it is expected that the walking cycle is regulated around the desired walking cycle $T_{\text {des }}$ after several steps. $T_{f f}$ is an initial value of $T_{s w}(k)$, and it does not affect the regulated walking cycle. In contrast with [15], this controller considers the deviation between the actual and desired walking cycle.

\subsection{Experimental Result}

An experiment was performed to confirm the validity of the designed feedback controller. In the experiment, the robot walked from the carpet floor to the linoleum floor while maintaining a certain desired walking cycle utilizing the feedback controller. Seeing from the Figure 7, we set $T_{\text {des }}$ as 1.15 [s] with which the robot can walk both on the carpet and linoleum floor. An estimated transition of the walking cycle $T(k)$ and the walking parameter $T_{s w}(k)$ are shown in Figure 8. This figure is produced from Figure 7 with the addition 


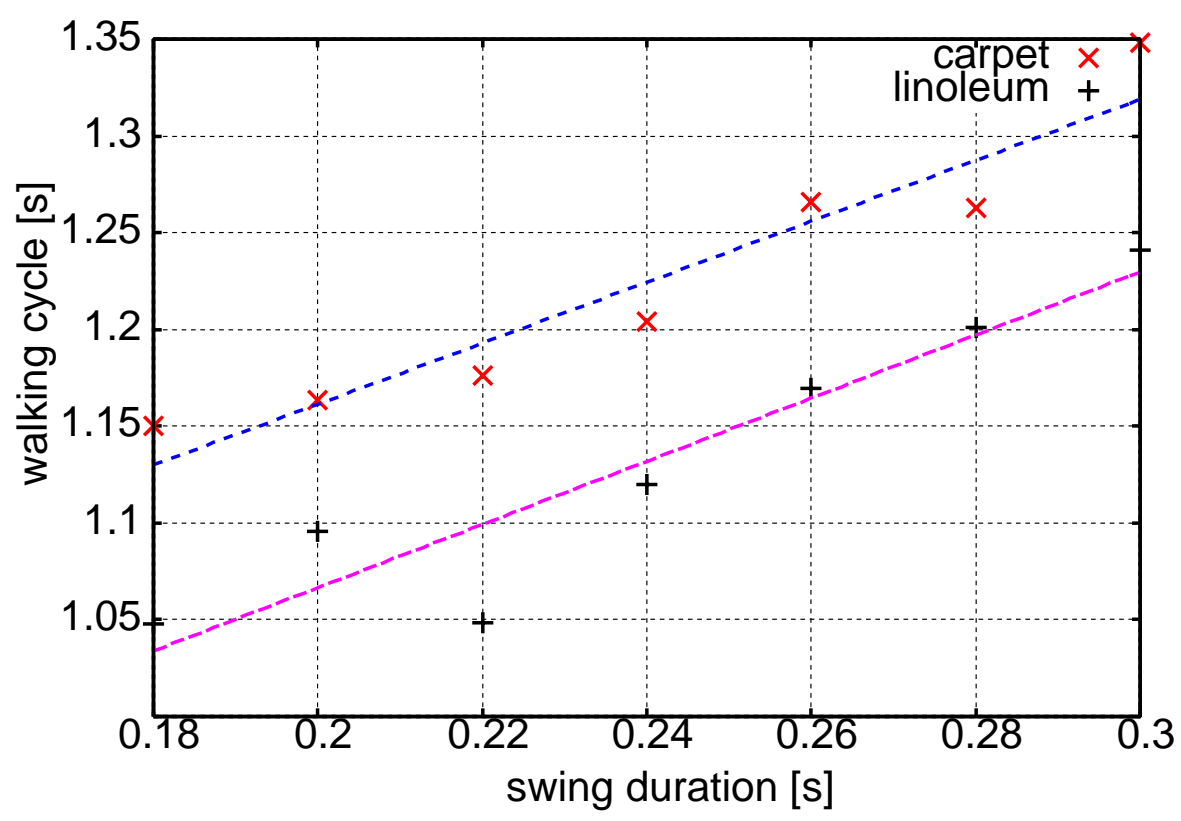

Figure 7: Relationship between swing duration and walking cycle on carpet and linoleum.

of the lines. As reported in [15], the walking cycle is changed when the robot walks over the small stair less than 10 [mm]. Therefore, we should prepare carpet and linoleum floor and let the height of the two floor surfaces be precisely same. However, it is technically difficult to let the surface be same. In order to show that the walking cycle is changed by the types of the floor, not by the stair, we allowed the robot walk on the linoleum floor and set $T_{s w}(0)=0.193$ with which the robot walks with desired walking cycle if the robot walks on the carpet floor. If the robot walks on the carpet floor with $T_{s w}(k)=0.193$, the robot walks with desired walking cycle (point "A" in Fig. 8) after several steps. However, the cycle is shorter (around 1.05 $[\mathrm{s}]$ ) than desired one (point "B" in Fig. 8) because the robot walks on the linoleum floor. We can detect the variation of the terrain by observing the walking cycle. When the walking cycle became shorter, the feedback controller (equation (3)) adjusted the walking parameter to maintain the walking cycle at 1.15 [s] (Fig. 8 "B" to "C"). In the equation (3), $T_{f f}$ was set to $T_{f f}=0.193[\mathrm{~s}]$.

The experimental results are shown in Figure 9. Figure 9(a) shows the walking cycle $T(k)$ and (b) shows the walking parameter $T_{s w}(k)$. In the figure, the parameter is fixed at $T_{s w}(k)=0.193[\mathrm{~s}]$ from the first to the fifth step. The walking cycle got shorter than the desired value (point "B" in Fig. 8). After the fifth step, the feedback controller began to regulate the walking cycle at $1.15[\mathrm{~s}]$. The feedback constants were set at $K_{p}=0.5$ and $K_{i}=0.5$. By regulating the walking cycle utilizing the feedback controller, the walking cycle lengthened, and the walking cycle approached the desired value after the eighth step (Point "B" to "C" in Fig. 8). As shown in Figure 9 (b), the walking parameter $T_{s w}(k)$ after the eighth step was close to $0.25[\mathrm{~s}]$, which corresponded to the value derived from equation (2). This result shows that the proposed feedback controller worked effectively to regulate the walking cycle. Note that it is hard to discuss a stability of walking because the property of the physical terrain is not uniformly same but different at every walker's landing point. We then regulate the walking cycle and the result shows that the walking cycle with the controller is within some range around the desired walking cycle.

\section{Conclusion}

In this paper, a biped walker that is driven by elastic artificial muscles was developed. The robot walked on various terrains, and the variation in the walking behavior as a result of the interaction between the robot dynamics and terrain was observed. In addition, the relationships between the walking parameter that corresponds to hip joint compliance and the walking cycle on various classes of the terrain were investigated and used to design a feedback controller that could regulate the walking cycle.

The variety of ground conditions seen in daily life is considerable. The goal of joint compliance is not only to realize adaptive locomotion by negotiating various terrains, but also to sense the classes of terrain. It is significant to build a physical robot that can walk on the ground because it is difficult to model the interaction between robot dynamics and the terrain.

This approach is effective only if the robot realizes adaptive locomotion. One of the future issues is to investigate the potential for adding another joint and for tuning the joint compliance in order to enlarge the 


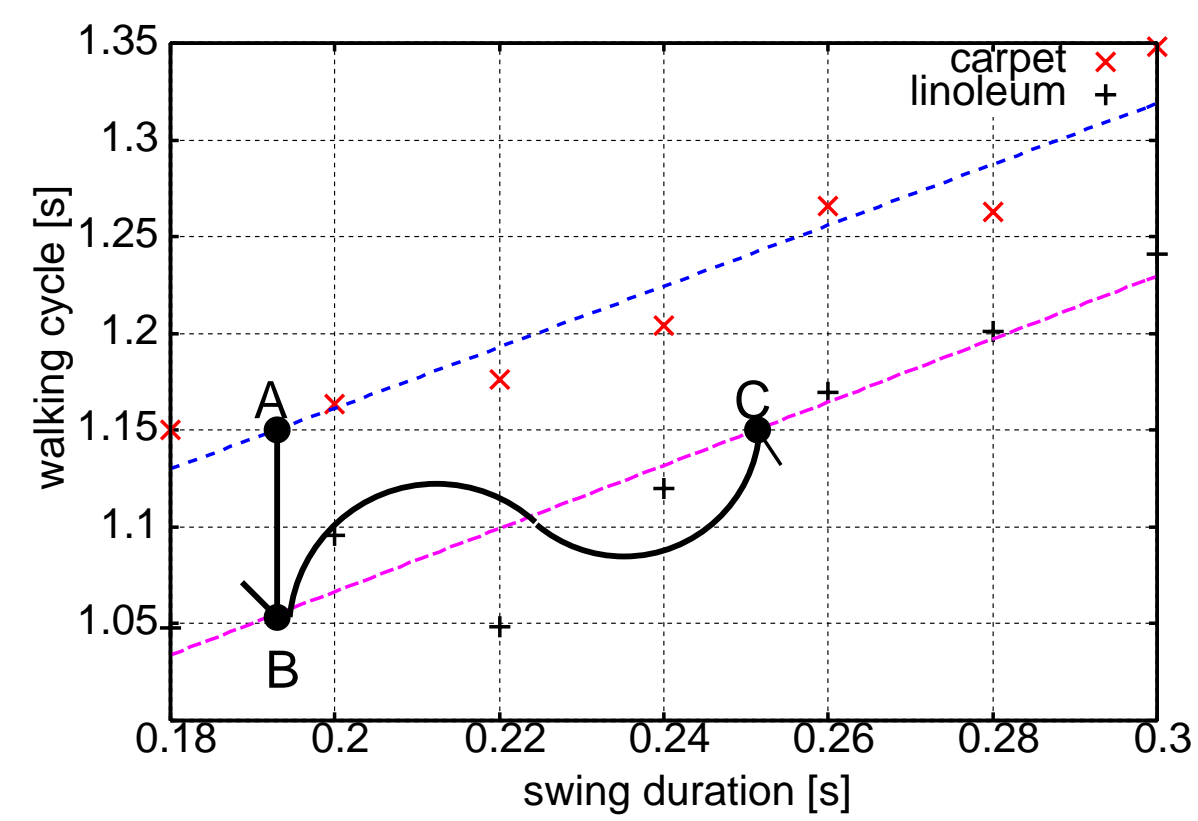

Figure 8: Relationship between the walking parameter and the walking cycle on carpet and linoleum. When the robot walks with a fixed walking parameter, the walking cycle becomes shorter when the robot walks from the carpet and the linoleum (line A-B), and $T_{s w}(k)$ should be adjusted in order to walk with the same walking cycle on different surfaces (curved line B-C)

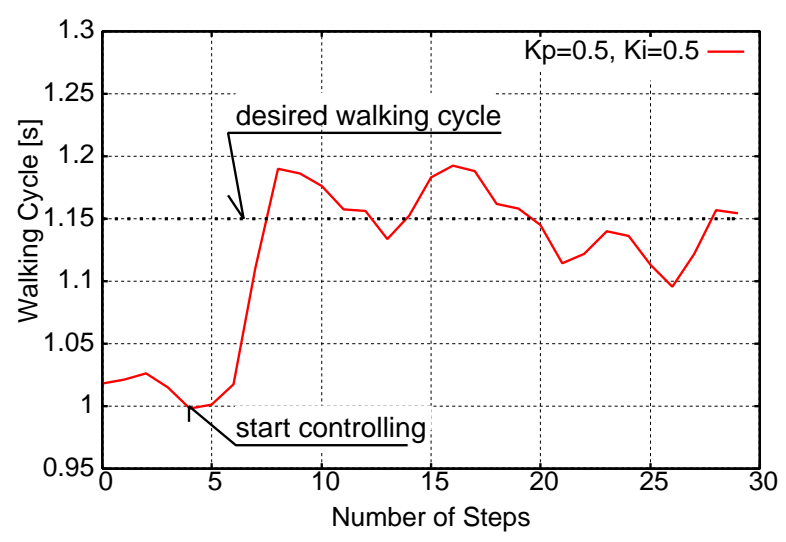

(a) walking cycle $T(k)$

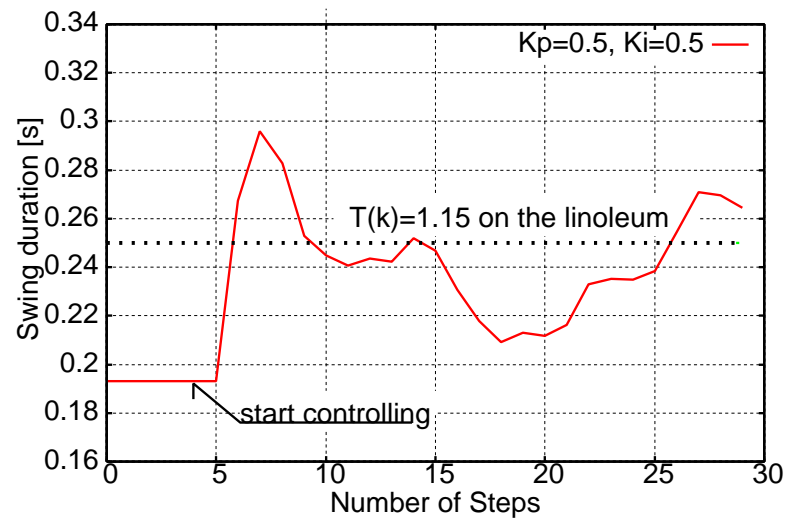

(b) Walking parameter $T_{s w}(k)$

Figure 9: Walking cycle and walking parameter when the robot walks from carpet to linoleum. The controller works after the fifth step, and $K p=0.5$ and $K i=0.5$. 
range of terrain class in which the robot can achieve adaptive locomotion. Compliant joints are a promising architecture not only for achieving adaptive locomotion, but also for sensing terrain.

\section{References}

[1] Y.S.Yoon and J.M.Mansour. The passive elastic moment at the hip. Journal of Biomechanics, Vol. 15, No. 12 , pp. 905-910, 1982.

[2] C.T.Farley and O.González. Leg stiffness and stride frequency in human running. Journal of Biomechanics, Vol. 29, No. 2, pp. 181-186, 1996.

[3] K.Akachi, K.Kaneko, N.Kanehira, S.Ota, G.Miyamori, M.Hirata, S.Kajita, and F.Kanehiro. Development of humanoid robot hrp-3p. In Proceedings of 2005 IEEE/RSJ International Conference on Humanoid Robots, pp. 50-55, 2005.

[4] I.Park, J.Kim, S.Park, and J.Oh. Development of humanoid robot platform khr-2. In IEEE-RAS/RSJ International Conference on Humanoid Robots (Humanoids), pp. CD-ROM, 2004.

[5] K. Hirai, M. Hirose, Y. Haikawa, and T. Takenaka. The development of honda humanoid robot. In Proceedings of the 1998 IEEE International Conference on Robotics and Automation (ICRA), pp. 13211326, 1998.

[6] T. McGeer. Passive dynamic walking. International Journal of Robotics Research, Vol. 9, No. 2, pp. 62-82, 1990.

[7] A. D. Kuo. Energetics of actively powered locomotion using the simples walking model. Journal of Biomechanical Engineering, Vol. 124, No. 1, pp. 113-120, February 2002.

[8] R.Q.van der Linde. Passive bipedal walking with phasic muscle contraction. Biological Cybernetics, Vol. 81, No. 3, pp. 227-237, 1999.

[9] M. Wisse and J. van Frankenhuyzen. Design and construction of mike ; 2d autonomous biped based on passeive dynamic walking. In 2nd International Symposium on Adaptive Motion of Animals and Machined (AMAM), 2002.

[10] C. P. Chou and B. Hannaford. Measurement and modeling of mckibben pneumatic artificial muscles. IEEE Transactions on Robotics and Automation, Vol. 12, No. 1, pp. 90-102, 1996.

[11] R. Q. van der Linde. Design, analysis, and control of a low power joint for walking robots, by phasic activation of mckibben muscles. IEEE Transactions on Robotics and Automation, Vol. 15, No. 4, pp. 599-604, 1999.

[12] HITACHI Medical corporation web page. http://www.hitachi-medical.co.jp/english/index.htm.

[13] Smc web page. http://www.smcworld.com.

[14] T.Takuma, S.Nakajima, M.Asada, and K.Hosoda. Design of self-contained biped walker with pneumatic actuators. In SICE Annual Conference, pp. CD-ROM, 2004.

[15] T.Takuma and K.Hosoda. Controlling the walking period of a pneumatic muscle walker. International Journal of Robotics Research, Vol. 25, No. 9, pp. 861-866, 2006. 